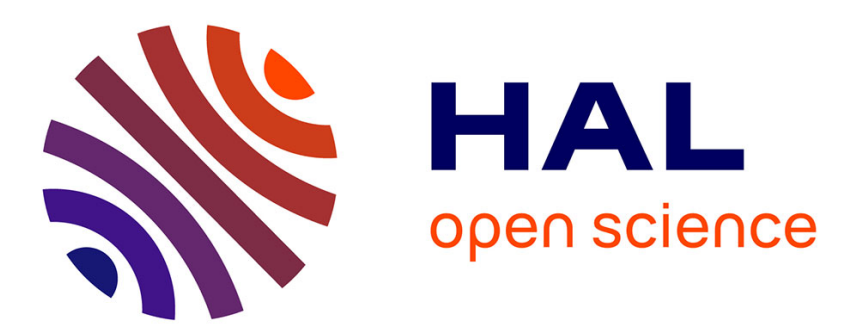

\title{
A linear model approach for ultrasonic inverse problems with attenuation and dispersion
}

Ewen Carcreff, Sébastien Bourguignon, Jérôme Idier, Laurent Simon

\section{To cite this version:}

Ewen Carcreff, Sébastien Bourguignon, Jérôme Idier, Laurent Simon. A linear model approach for ultrasonic inverse problems with attenuation and dispersion. IEEE Transactions on Ultrasonics, Ferroelectrics and Frequency Control, 2014, 61 (7), pp.1191-1203. 10.1109/TUFFC.2014.3018 . hal01005337

\section{HAL Id: hal-01005337 \\ https://hal.science/hal-01005337}

Submitted on 12 Jun 2014

HAL is a multi-disciplinary open access archive for the deposit and dissemination of scientific research documents, whether they are published or not. The documents may come from teaching and research institutions in France or abroad, or from public or private research centers.
L'archive ouverte pluridisciplinaire HAL, est destinée au dépôt et à la diffusion de documents scientifiques de niveau recherche, publiés ou non, émanant des établissements d'enseignement et de recherche français ou étrangers, des laboratoires publics ou privés. 


\title{
A linear model approach for ultrasonic inverse problems with attenuation and dispersion
}

\author{
Ewen Carcreff, Sébastien Bourguignon, Jérôme Idier, Member, IEEE, and Laurent Simon, Member, IEEE
}

\begin{abstract}
Ultrasonic inverse problems such as spike train deconvolution, synthetic aperture focusing or tomography aim to reconstruct spatial properties of an object (discontinuities, delaminations, flaws, etc.) from noisy and incomplete measurements. They require an accurate description of the data acquisition process. Dealing with frequency-dependent attenuation and dispersion is therefore crucial since both phenomena modify the wave shape as the travel distance increases. In an inversion context, this paper proposes to exploit a linear model of ultrasonic data taking into account attenuation and dispersion. The propagation distance is discretized in order to build a finite set of radiation impulse responses. Attenuation is modeled with a frequency power law and then dispersion is computed in order to yield physically consistent responses. Using experimental data acquired from attenuative materials, this model outperforms the standard attenuation-free model and other models of the literature. Due to model linearity, robust estimation methods can be implemented. When matched filtering is employed for single echo detection, the model that we propose yields precise estimation of the attenuation coefficient and of the sound velocity. A thickness estimation problem is also addressed through spike deconvolution where the proposed model also achieves accurate results.
\end{abstract}

\section{INTRODUCTION}

Ultrasonic waves are widely used for non destructive testing (NDT) of materials [1,2], tissue characterization [3] and biomedical imaging $[4,5]$. Many applications in these fields can be formulated as inverse problems such as spike train deconvolution [6-8], biomedical image restoration [4, 9], timeof-flight tomography [10-12] and synthetic aperture focusing techniques (SAFT) [13-15]. Such problems rely on both an accurate direct model describing the acquisition process and appropriate prior information constraining the solution [8].

Attenuation and dispersion can arise in the applications cited above. Attenuation is due to two basic causes, namely, scattering and absorption. Scattering results from the fact that the material is not strictly homogeneous, implying multiple direction propagation. Absorption is caused by the excitation of the particles that converts sound energy into heat. Consequently, attenuation increases with frequency. Most models in the literature consider a frequency power law $[3,16]$. This frequency-dependent loss has a low-pass filtering effect on the transmitted waves and grows as propagation distance increases. It generates a shape broadening of the echoes that degrades the resolution [17]. Dispersion - a by-product of attenuation - means that the phase velocity depends on frequency, producing a frequency-dependent phase variation of the echoes [5, 17, 18].

Attenuation and dispersion are often overlooked in ultrasonic direct models for inversion purposes. Most formulations consider a linear model that is invariant with respect to the propagation distance. However, if the ultrasonic propagation characteristics are not considered, the performance of the algorithms can be degraded because the model accuracy is too weak. Several approaches have been developed to include attenuation in acoustical inverse problems, mostly in geophysics [19-21]. Indeed, similar effects impact the propagation of seismic waves that is modeled as the $Q$-filter $[19,22]$. In the field of ultrasonic NDT, some methods overcome the issue of wave distortion by proposing a greater flexibility in the direct model. On the one hand, parametric methods associate a specific shape to each echo, which is usually modeled as a modulated Gaussian pulse. The parameters of each echo are then estimated by non-linear least-squares fitting [23] or by greedy procedures such as matching pursuit [24-26]. On the other hand, non-parametric approaches employ a blind strategy where weaker constraints are imposed on the echo shape - as, for example, slow variations between neighboring intervals of the propagation distance [27]. Even if such models allow for some shape variation of the echoes with respect to the propagation distance, they do not introduce any physical knowledge about the propagation properties.

The present paper aims at contributing to the solving of ultrasonic inverse problems by including attenuation and dispersion in the direct model. In particular, we propose to account for physical attenuation profiles defined in the frequency domain such as power law attenuation models. Our objective is threefold. First, we improve the ultrasound model accuracy compared to the standard attenuation-free model. Second, in contrast with the methods described above [23-27], we yield a more constrained description of the data. Consequently, better performance of the inversion procedure is expected. In particular, a more accurate model aims at improving echo detection for long propagation distances where the signal-to-noise ratio is low. Last, our framework yields a linear direct model which enables the use of many acknowledged inversion methods [8]. Related works [7,28] proposed similar approaches but with an empirical description of attenuation within a time-domain signal model. The model that we propose is derived from the physics of wave propagation and is described in the frequency domain $[3,16]$.

In this paper, the signal model is formulated as a set of transfer functions in the Fourier domain [29]. The propagation in the medium is modeled by a so-called radiation transfer function [30] depending on the wavenumber $k(f)=\beta(f)-j \alpha(f)$. The term $\beta(f)$ is related to the phase velocity and $\alpha(f)$ is the attenuation. To ensure the consistency of the corresponding radiation impulse response, 
$\alpha(f)$ and $\beta(f)$ are analytically linked by conditions derived from the Kramers-Kronig relations [31]. Several models have been developed, both for continuous-time and discrete-time signals, and validated for materials having linear and non linear attenuation [31-34]. In this paper, we will use the formulation proposed by Kuc [35,36]. As attenuation depends on propagation distance, we build a set of radiation impulse responses by an appropriate discretization of the space domain. We then obtain a discrete linear model of data $\boldsymbol{y}=\mathbf{H}_{e} \mathbf{H}_{a} \boldsymbol{x}$. The matrix $\mathbf{H}_{e}$ represents the instrumental response, which is invariant with respect to the propagation distance. The matrix $\mathbf{H}_{a}$ stands for the set of radiation impulse responses. The vector $\boldsymbol{x}$ represents the unknown spatial distribution of targets. Note that standard direct models consider an invariant model with respect to the propagation distance, that is, $\boldsymbol{y}=\mathbf{H}_{e} \boldsymbol{x}$.

A major advantage of the obtained model holds in its generality for a large variety of ultrasonic inverse problems. The purpose of inversion is then to estimate the object $\boldsymbol{x}$ based on the knowledge of $\boldsymbol{y}, \mathbf{H}_{e}$ and $\mathbf{H}_{a}$, and on some prior information on $\boldsymbol{x}$. In NDT for example, $\boldsymbol{x}$ is expected to have a few non-zero elements, corresponding to the positions of impedance discontinuities including flaws [6]. The description and the validation of sophisticated inversion algorithms are out of the scope of this paper. Consequently, experiments are dedicated to rather simple estimation problems in homogeneous plates. We use two basic inverse methods, namely, a matched filtering procedure and an $\ell_{1}$-norm-based spike train deconvolution method [6] applied to non-overlapping echoes.

The paper is organized as follows. Section II describes the model of ultrasonic signals, defined in the frequency domain. The relation proposed by Kuc [35] between the phase and the magnitude of the radiation transfer function is detailed. The model is validated on experimental data acquired in a polymethyl methacrylate (PMMA) plate. Then, in Section III, a linear direct model is built, based on the discretization of the unknown spatial source distribution. Such a frequency-based approach is compared with Olofsson's time-domain model [7]. In Section IV, experimental results are shown through the non destructive evaluation of a polycarbonate plate in order to estimate the attenuation coefficient and the velocity. Section V considers a typical plate thickness estimation problem where a spike train deconvolution method is considered using our model. The paper ends with a discussion in Section VI.

\section{Propagation MOdel of Ultrasonic Signals}

\section{A. Model for a single target}

Here, the ultrasonic measurement is made in a homogeneous and isotropic medium containing a single point-like target placed at spatial location $\boldsymbol{r}_{T}$. The configuration can either use two transducers, a transmitter and a receiver (T/R), or a single transducer in pulse/echo (P/E) mode - see Figure 1 for the description of both configurations. The received signal can be defined in the Fourier domain by a set of transfer functions [29, 37]:

$$
Y\left(f, \boldsymbol{r}_{T}\right)=U(f) H_{e a}(f) H_{r}\left(f, \boldsymbol{r}_{T}\right) H_{a e}(f),
$$

as illustrated in Figure 2. $U(f)$ is the electrical excitation pulse

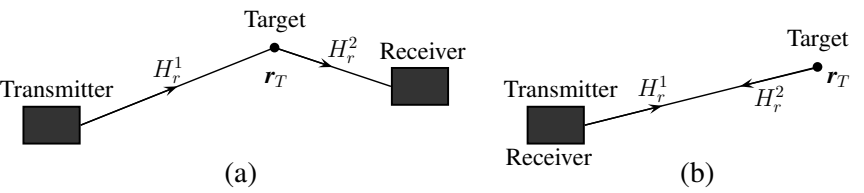

Fig. 1. Two possible configurations for ultrasonic data aquisition: (a) Transmit/Receive mode and (b) Pulse/Echo mode.

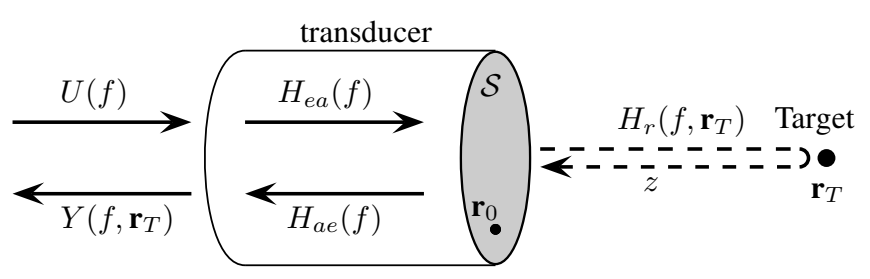

Fig. 2. Pulse-echo measurement for a target located at $\boldsymbol{r}_{T}$ using a radiating surface $\mathcal{S}$. The received signal $Y\left(f, \boldsymbol{r}_{T}\right)$ is modeled through a set of transfer functions: $Y\left(f, \boldsymbol{r}_{T}\right)=U(f) H_{e a}(f) H_{r}\left(f, \boldsymbol{r}_{T}\right) H_{a e}(f) . z$ is the overall propagation distance.

sent to the emitting transducer. The functions $H_{e a}(f)$ and $H_{a e}(f)$ are the electro-acoustical and acousto-electrical frequency responses of the transducers, respectively. The global instrumental function $H_{e}(f)=U(f) H_{e a}(f) H_{a e}(f)$ can be defined by collecting the functions that do not depend on the propagation in the material. The received spectrum is hence:

$$
Y\left(f, \boldsymbol{r}_{T}\right)=H_{e}(f) H_{r}\left(f, \boldsymbol{r}_{T}\right) .
$$

The radiation transfer function $H_{r}\left(f, \boldsymbol{r}_{T}\right)$ represents the transfer function related to the propagation path $[29,30]$. As an example, let us consider the one-way path from the emitting transducer with surface $S$ to the target. The radiation transfer function ${ }^{1}$ in $\boldsymbol{r}_{T}$ is the sum over elementary contributions of sources over the surface $S$ [30]:

$$
H_{r}^{1}\left(f, \boldsymbol{r}_{T}\right)=\int_{\boldsymbol{r}_{0} \in S} \frac{e^{-j k(f)\left\|\boldsymbol{r}_{T}-\boldsymbol{r}_{0}\right\|}}{2 \pi\left\|\boldsymbol{r}_{T}-\boldsymbol{r}_{0}\right\|} \mathrm{d} S .
$$

In T/R mode, as represented in Figure 1, the overall radiation transfer function is the product of two different radiation functions (transmitter to target and target to receiver), whereas in P/E mode, the two transfer functions are equal [38]. Note that one could consider specific target surfaces, leading to different reflector signatures [37]. For example, Lhémery has developed a model with small oriented targets, including the diffraction of the transducers [38]. In the current paper, we consider targets with identical signatures.

The complex-valued frequency-dependent wavenumber $k(f)$ can be written:

$$
k(f)=\beta(f)-j \alpha(f) .
$$

The term $\beta(f)$ describes the propagation of the wave such that $\beta(f)=2 \pi f / c(f)$, with $c(f)$ the phase velocity. The term $\alpha(f)$ represents the attenuation in the material. By neglecting the diffraction of the transducers, we assume that

\footnotetext{
${ }^{1}$ By convention, for a frequency $f$, we consider a plane wave $e^{j(2 \pi f t-k(f) x)}$ propagating in the positive $x$ direction, where $k(f)$ is the wavenumber.
} 
the distance of propagation is roughly constant for all points of the radiating and receiving surfaces [29]. Let $z$ represent the distance of the travel path from the center of the emitter to the center of the receiver through the target. From (3) and (4), one can write the radiation transfer function:

$$
\begin{aligned}
H_{r}(f, z) & =b(z) e^{-\alpha(f) z} e^{-j \beta(f) z} \\
& =b(z) e^{-\alpha(f) z} e^{-j 2 \pi f z / c(f)} .
\end{aligned}
$$

The frequency-independent term $b(z)$ depends on the propagation distance and on several factors such as the transducer and target surfaces and their relative positions. For each $z$, the radiation impulse response $h_{r}(t, z)$ is given by the inverse Fourier transform of $H_{r}(f, z)$. Similarly, $h_{e}(t)$ is the impulse response corresponding to $H_{e}(f)$. From (2), the time-domain signal received by the transducer for a single target is:

$$
y(t, z)=h_{e}(t) * h_{r}(t, z),
$$

which is the convolution between the instrumental impulse response $h_{e}(t)$ and the radiation impulse response $h_{r}(t, z)$ depending on the propagation distance $z$.

Most ultrasound propagation models in tissue characterization and NDT consider a frequency power law attenuation model [16]:

$$
\alpha(f)=\alpha_{0}|f|^{\gamma},
$$

where $\alpha_{0}$ and $\gamma$ are real positive parameters characterizing a given material. Generally, the frequency power parameter satisfies $1 \leq \gamma \leq 2[16,34]$. For tissues, one has typically $1 \leq \gamma \leq 1.5$ [39]. Many materials have linear attenuation, that is, $\gamma=1[3,32]$. Numerous methods have been proposed to measure the parameter $\alpha_{0}$ in such a case [3,35, $36,40]$. In NDT, $\gamma>1$ corresponds to non-linear attenuation and is related to more complex material structures, for instance polyethylene $(\gamma \approx 1.13)$, synthetic rubber $(\gamma \approx 1.38)$ or castor oil $(\gamma \approx 1.67)[34,40]$.

Note that the attenuation model (7) is similar to the constant$Q$ model employed in geophysics for seismic waves [22]. $Q$ is a quality factor and can be defined as $Q=\pi / \alpha_{0} c_{0}$, if $c_{0}$ is the constant wave velocity [21]. The parameter $Q$ is also inversely proportional to $\alpha_{0}$, meaning that infinite $Q$ corresponds to a lossless medium $\left(\alpha_{0}=0\right)$. Similarly, it has been shown that propagation in soils is adequately modeled with $\gamma \in[1,2]$ [21].

From (5) and (7), attenuation has a low-pass effect, causing a downshift of the center frequency of the echoes as the propagation distance increases $[16,17,41]$ and limiting the resolution. Such effect is represented in Figure 3 a which shows a typical radiation transfer function $\left|H_{r}(f, z)\right|$ as a function of both frequency $f$ and propagation distance $z$. This implies the use of relatively low-frequency transducers and a strong amplitude loss for high distances. In the time domain, this effect causes a broadening of the echoes that lessens the resolution as the distance increases.

\section{B. Causality of the radiation impulse response}

For physical reality purpose, constraints are applied on the radiation impulse response $h_{r}(t, z)$. First of all, the response is real-valued and therefore implies the Hermitian symmetry property $H_{r}(-f, z)^{*}=H_{r}(f, z)$, where superscript * stands for complex conjugation. As a consequence, according to (4) and (5), the wavenumber has the anti-Hermitian symmetry property, leading to $\alpha(f)$ even and $\beta(f)$ odd. Secondly, in acoustics, the phase velocity $c(f)$ increases as a function of frequency, which is called the anomalous dispersion [34]. Therefore, there exists a maximum velocity for $f=\infty$ [42], say, $c_{0}$. For a given distance $z$, this maximum velocity is directly linked to a minimum time of flight $t_{0}=z / c_{0}$ such that $h_{r}(t, z)=0$ for $t<t_{0}$. In other words, an ultrasonic wave emitted at $t=0$ should not appear before $t_{0}$ for a target located at distance $z$. Note that $c_{0}$ is larger than the group velocity which is the velocity of the envelope of the waveform. Such causality principle implies specific relations between the phase and the magnitude in (5) $[34,43]$.

Kak and Dines [3] proposed a linear phase model, under a linear attenuation assumption $\alpha(f)=\alpha_{0}|f|$, by considering a constant phase velocity $c(f)=c_{0}$ :

$$
H_{r}(f, z)=b(z) e^{-\alpha(f) z} e^{-j 2 \pi f z / c_{0}} .
$$

In this case, $c_{0}$ also corresponds to the group velocity. The inverse Fourier transform of $e^{-j 2 \pi f z / c_{0}}$ is a delta function $\delta\left(t-z / c_{0}\right)$ corresponding to a pure delay of $t_{0}=z / c_{0}$. However, under this linear phase assumption, the impulse response $h_{r}(t, z)$ is symmetric with respect to $t_{0}$ and hence is not causal [3].

Indeed, the Paley-Wiener condition states that $h_{r}(t, z)$ is causal if and only if [44]:

$$
\int_{-\infty}^{+\infty} \frac{|\ln | H_{r}(f, z)||}{1+f^{2}} \mathrm{~d} f<\infty .
$$

In such a case, the corresponding phase term is derived from the Kramers-Kronig relations [31,44]. For the power law attenuation model (7), equation (9) is verified only for $\gamma<1$ [40, $42,43]$, that is, $h_{r}(t, z)$ is not causal for $\gamma \geq 1$.

Gurumurthy and Arthur [32] considered a minimum-phase model $[44,45]$ accounting for dispersion in the case $\gamma=1$. They considered that attenuation grows only sub-linearly at high frequencies in order to compute the dispersion from the Kramers-Kronig relations [32]. Nevertheless, this model is not strictly causal since the Paley-Wiener condition is not respected. A dispersion term $\epsilon(f)$ is added to the linear phase term in (8), which yields:

$$
H_{r}(f, z)=b(z) e^{-\alpha(f) z} e^{-j 2 \pi f z / c_{0}} e^{-j \epsilon(f) z} .
$$

$H_{r}(f, z)$ can then be separated into the linear-phase function $b(z) e^{-j 2 \pi f z / c_{0}}$ and the attenuation function:

$$
H_{a}(f, z)=e^{-\alpha(f) z} e^{-j \epsilon(f) z},
$$

that is, in the time domain:

$$
\begin{aligned}
h_{r}(t, z) & =b(z) h_{a}(t, z) * \delta\left(t-z / c_{0}\right) \\
& =b(z) h_{a}\left(t-z / c_{0}, z\right) .
\end{aligned}
$$

with $h_{a}(t, z)$ the attenuation impulse response corresponding to $H_{a}(f, z)$.

Analogously, Kuc derived a minimum-phase model for discrete-time signals for $\gamma=1[35,36]$, that can be easily 
(a)

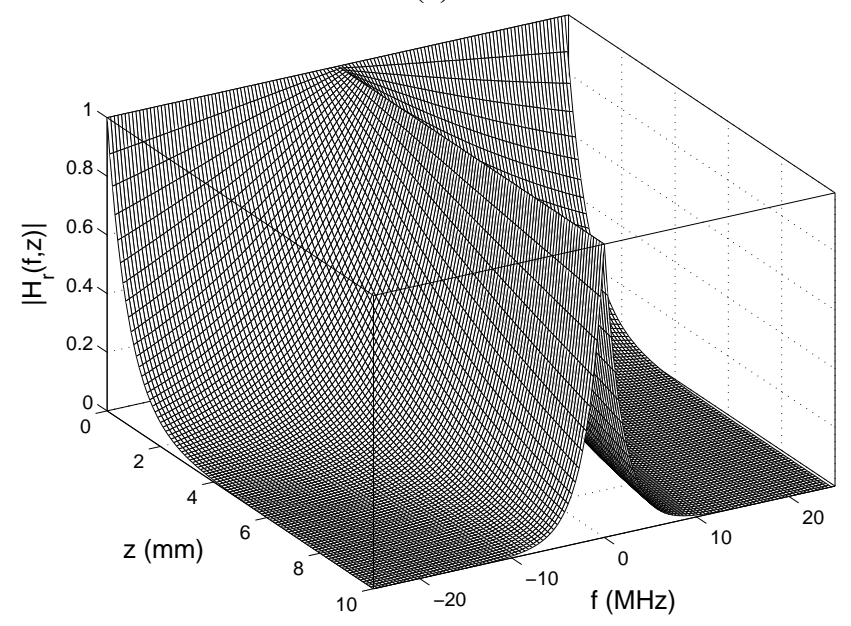

(b)

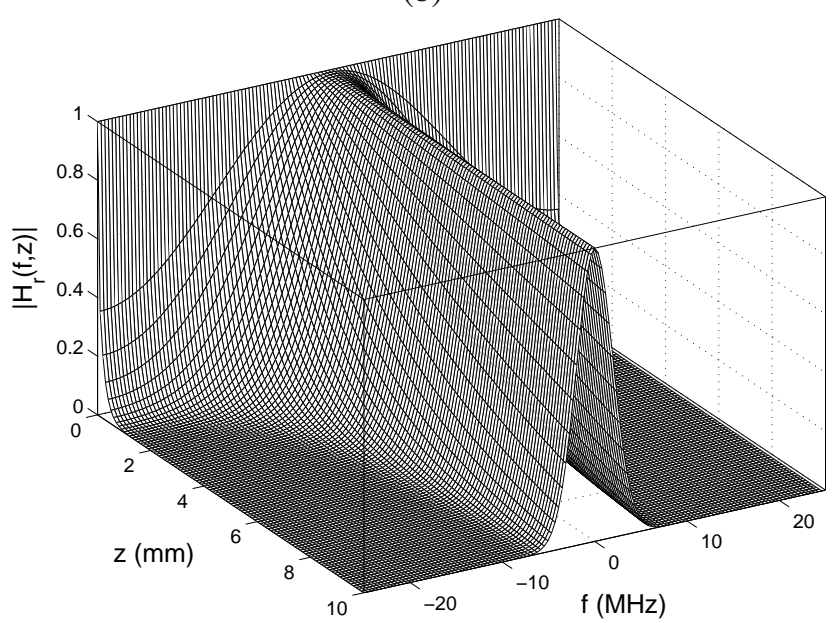

Fig. 3. Examples of attenuation as a function of frequency $f$ and of propagation distance $z$. The larger the distance, the more important the low-pass filtering effect of attenuation. (a): Linear attenuation model: $\left|H_{r}(f, z)\right|=e^{-\alpha_{0}|f| z}$, with $\alpha_{0}=50 \mathrm{~Np} / \mathrm{MHz} / \mathrm{m}$. (b): Olofsson's model [7] with $a=0.2$ (see III-C).

extended to non-linear attenuation. In this paper, we will use this formulation to describe $H_{a}(f, z)$, which is detailed hereafter.

\section{Causality constraint on the discrete-time impulse response}

For continuous-time signals, the causality of the system defined by the transfer function $H(f)=H_{R}(f)+j H_{I}(f)$ imposes that $H_{I}$ is the Hilbert transform of $H_{R}$ [44]. The equivalent characterization for discrete-time signals reads [45]:

$$
H_{I}(f)=-\frac{1}{f_{S}} \mathcal{P} \int_{-\frac{f_{S}}{2}}^{\frac{f_{S}}{2}} H_{R}(g) \cot \left(\frac{\pi}{f_{S}}(f-g)\right) \mathrm{d} g,
$$

where $f_{S}$ is the sampling frequency and $\mathcal{P}$ denotes the Cauchy principal value of the integral. Taking the logarithm of $H_{a}(f, z)$ in (11): $\ln H_{a}(f, z)=-\alpha(f) z-j \epsilon(f) z$ and assuming that the corresponding impulse response is causal [45] leads to:

$$
\epsilon(f)=\mathcal{H}(\alpha(f)),
$$

where $\mathcal{H}(\alpha(f))$ is defined by:

$$
\mathcal{H}(\alpha(f))=-\frac{1}{f_{S}} \mathcal{P} \int_{-\frac{f_{S}}{2}}^{\frac{f_{S}}{2}} \alpha(g) \cot \left(\frac{\pi}{f_{S}}(f-g)\right) \mathrm{d} g .
$$

Note that this expression is well-defined for a large class of attenuation models $\alpha(f)$. Moreover, $\mathcal{H}(\alpha(f))$ has an analytic expression for linear attenuation as established in Appendix, which is useful for fast and precise computations. For power law attenuation models with $\gamma \neq 1$, (15) can be computed by numerical integration.

The dispersion term (15) has been used in the case of linear attenuation to model attenuated signals in PMMA plates [35, 36]. Several studies precisely compared phase velocities from measured signals with the model in (14) [40,43]. They concluded to a satisfactory agreement from materials having linear and non-linear attenuation (PMMA, rubber, castor oil).

A synthetic example of impulse responses $h_{r}(t, z)$ is presented in Figure 4. A single target is located at dis-

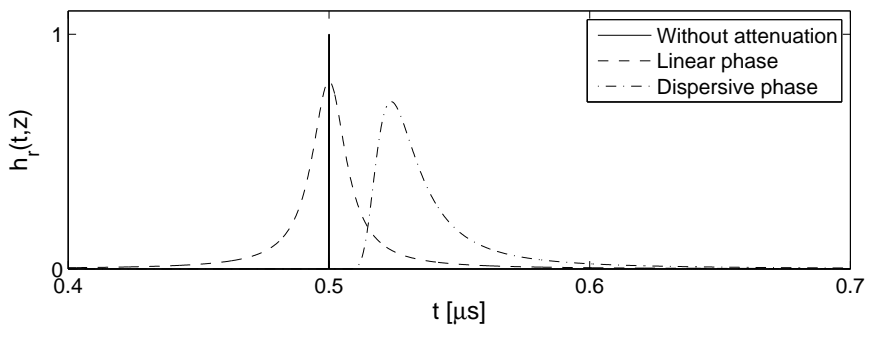

Fig. 4. Simulated impulse responses $h_{r}(t, z)$ using three propagation models: without attenuation, with linear phase and with dispersive phase. Parameters: $c_{0}=2000 \mathrm{~m} / \mathrm{s}, \gamma=1, \alpha_{0}=50 \mathrm{~Np} / \mathrm{MHz} / \mathrm{m}, z=1 \mathrm{~mm}$ and $b(z)=1$. The minimum time of flight is then $t_{0}=z / c_{0}=0.5 \mu \mathrm{s}$. This value corresponds to: (1) the position of the delta function $\delta\left(t-t_{0}\right)$, (2) the center of the linear-phase impulse response and (3) the starting time of the dispersive-phase impulse response.

tance $z=1 \mathrm{~mm}$ in a homogeneous and isotropic material with propagation parameters $c_{0}=2000 \mathrm{~m} / \mathrm{s}, \quad \gamma=1$, $\alpha_{0}=50 \mathrm{~Np} / \mathrm{MHz} / \mathrm{m}$ and $b(z)=1$. Three propagation models are used: without attenuation, with linear phase (8) and with dispersive phase (14). The response without attenuation is a delta function with a shift of $t_{0}=z / c_{0}=0.5 \mu \mathrm{s}$. The impulse response of the linear phase model has a symmetric shape on both sides of $t_{0}$, that violates the causality condition. As expected, the impulse response of the dispersive model appears to be causal since the values are very close to zero - less than $10^{-10}$ - before $t_{0}$.

\section{Validation with experimental data}

We now assess the accuracy of the dispersive model and compare it with other available models. The measurements are performed using a flat circular transducer of diameter $12.7 \mathrm{~mm}$ and center frequency $2.25 \mathrm{MHz}$. The object under test is a $25 \mathrm{~mm}$-thick PMMA plate, immersed in a water tank as illustrated in Figure 5. The plate is in the far field of the transducer and with normal incidence. Data are acquired at sampling frequency $f_{S}=100 \mathrm{MHz}$ and averaged over 100 


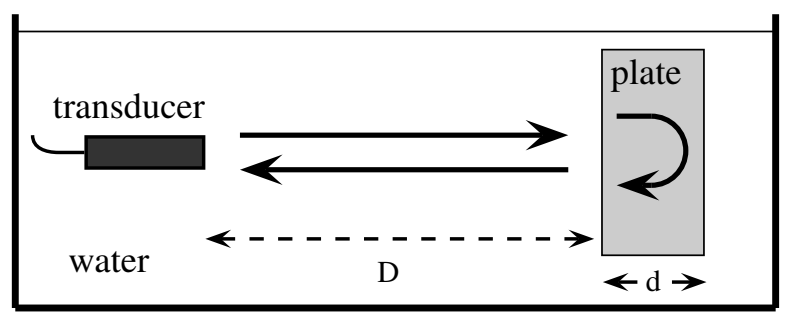

Fig. 5. Simplified data measurement setup. The plate of thickness $d$ is placed normally in the far field of the transducer.

realizations in order to reduce the noise level. We extract the two significant echoes from the data: the frontwall echo $y_{f}(t)$ (Figure 6a) and the backwall echo $y_{b}(t)$ (Figure 6b). Their respective Fourier transforms, say $Y_{f}(f)$ and $Y_{b}(f)$, are plotted in moduli in Figure 6c, showing a strong amplitude loss and a frequency downshift between the frontwall and the backwall echoes.

From (2) and (5), one has:

$$
Y_{f}(f)=b(2 D) H_{e}(f) e^{-\left(\alpha_{w}(f)+j \beta_{w}(f)\right) 2 D},
$$

where $b(2 D)$ is the reflection coefficient at the front face of the plate [46]. The terms $\alpha_{w}(f)$ and $\beta_{w}(f)$ stand for the propagation parameters in water (see (4)). The backwall echo is modeled as:

$Y_{b}(f)=b(2 D+2 d) H_{e}(f) e^{-\left(\alpha_{w}(f)+j \beta_{w}(f)\right) 2 D-(\alpha(f)+j \beta(f)) 2 d}$

with $b(2 D+2 d)$ the resulting amplitude for the whole wave travel. Dividing $Y_{b}(f) / Y_{f}(f)$ enables the cancellation of $H_{e}(f)$ and of the terms related to the propagation in water:

$$
\frac{1}{2 d} \ln \left(\frac{\left|Y_{b}(f)\right|}{\left|Y_{f}(f)\right|}\right)=\alpha(f)+\frac{1}{2 d} \ln \left|\frac{b(2 D+2 d)}{b(2 D)}\right| .
$$

Figure $6 \mathrm{~d}$ shows that such a function is approximately linear with respect to frequency, say $\alpha(f) \simeq \alpha_{0}|f|$. A linear regression leads to $\alpha_{0} \simeq 11.55 \mathrm{~Np} / \mathrm{MHz} / \mathrm{m}$ that is coherent with the values proposed in the literature $[40,47]$.

By neglecting attenuation and dispersion in water, which is usually assumed in the literature [40], one has $\alpha_{w}(f)=0$ and $\beta_{w}=2 \pi f / c_{w}$ where $c_{w}$ is the constant speed of sound in water. Therefore, the frontwall echo is:

$$
Y_{f}(f)=b(2 D) H_{e}(f) e^{-j 2 \pi f 2 D / c_{w}}
$$

in the frequency domain, that is, in the time domain: $y_{f}(t)=b(2 D) h_{e}\left(t-2 D / c_{w}\right)$. According to (17), we then fit the backwall echo $y_{b}(t)$ with

$$
\widehat{y}_{b}(t)=b(2 D+2 d) h_{e}\left(t-2 D / c_{w}\right) * h_{r}(t, 2 d),
$$

where $h_{e}\left(t-2 D / c_{w}\right)$ is obtained from $y_{f}(t)$ and $h_{r}(t, 2 d)$ is set from the three models introduced in II-B: without attenuation, with linear phase as in (8) and with dispersive phase as in (14). In the two last cases, attenuation is supposed linear, with $\alpha_{0}$ at the previously estimated value. The velocity $c_{0}$ is calculated from $2 d /\left(t_{b}-t_{f}\right)$ where $t_{f}$ and $t_{b}$ stand for the frontwall and backwall times of flight, respectively, yielding $c_{0} \approx 2802 \mathrm{~m} / \mathrm{s}$. For each model, the amplitudes and the times (a) Frontwall echo $y_{f}(t)$

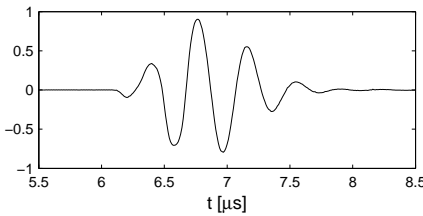

(c) Spectral magnitudes

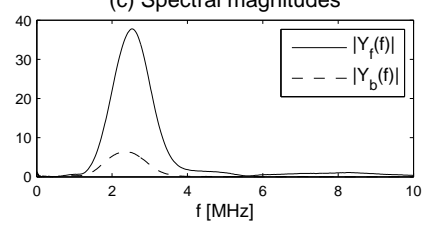

(e) Without attenuation
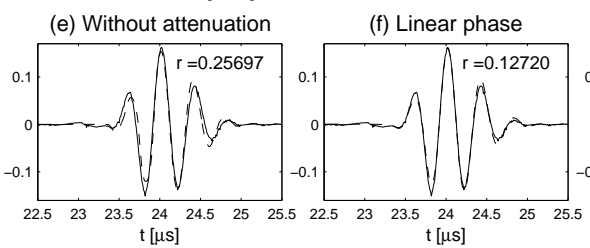

(b) Backwall echo $y_{b}(t)$

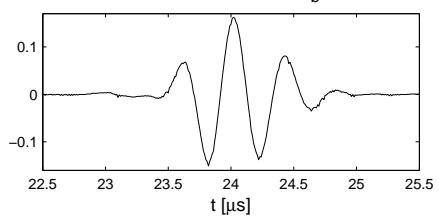

(d) Attenuation measurement

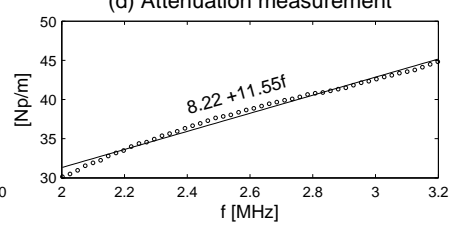

(g) Dispersive phase

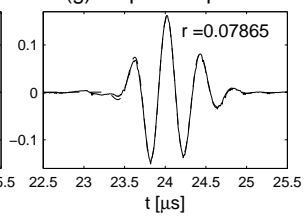

Fig. 6. Attenuation and dispersion models for data acquired from a PMMA plate with thickness $\mathrm{d}=25 \mathrm{~mm}$ using a $2.25 \mathrm{MHz}$ probe. (a): frontwall and (b) backwall echoes. (c): Magnitude spectra $\left|Y_{f}(f)\right|$ (solid line) and $\left|Y_{b}(f)\right|$ (dashed line). (d): Spectral magnitude ratio $\frac{1}{2 d} \ln \left(\frac{\left|Y_{b}(f)\right|}{\left|Y_{f}(f)\right|}\right)$ (circles) and linear regression (solid line). (e-f-g): Measured backwall echo (solid line) and three different models (dashed line), with $r$ the quadratic error between data and model. Parameters : $c_{0}=2802 \mathrm{~m} / \mathrm{s}, \alpha_{0}=11.55 \mathrm{~Np} / \mathrm{MHz} / \mathrm{m}, \gamma=1$ (linear attenuation assumption).

of flight of the echoes are optimized in order to achieve the best least-squares fit. The results are plotted in panels e-f$\mathrm{g}$ of Figure 6. The quadratic error between the data $y_{b}(t)$ and the model $\widehat{y}_{b}(t)$ is also computed and displayed on the corresponding subfigures. As expected, the dispersive model gives the best results, followed by the linear phase model.

\section{A DISCRETE-TIME LINEAR MODEL FOR INVERSE PROBLEMS}

\section{A. Inversion framework}

Let us consider a propagation medium composed of an unknown distribution of point-like targets. From (6) and (12), the received signal is then the sum over all the target contributions:

$$
\begin{aligned}
y(t) & =\int_{z} h_{e}(t) * h_{r}(t, z) \mathrm{d} z \\
& =\int_{z} b(z) h_{e}(t) * h_{a}\left(t-z / c_{0}, z\right) \mathrm{d} z \\
& =\int_{z} b(z)\left(\int_{u} h_{e}(u) h_{a}\left(t-u-z / c_{0}, z\right) \mathrm{d} u\right) \mathrm{d} z
\end{aligned}
$$

where $b(z)$ describes the material spatial content independently of the ultrasonic wave propagation effects. In this context, inversion aims at reconstructing such a function from a finite number of noisy samples of $y(t)$. The reconstruction procedure might incorporate some prior knowledge on $b(z)$. For example, from an acquired A-scan in NDT, $b(z)$ may be a spike train containing the spatial positions of the acoustical impedance changes [7]:

$$
b(z)=\sum_{k=1}^{K} b_{k} \delta\left(z-z_{k}\right),
$$


where unknown parameters $z_{k}$ and $b_{k}$ represent the position of the $k$-th impedance discontinuity and the associated amplitude, respectively. In the case where no attenuation is taken into account, that is $h_{a}(t, z)=\delta(t)$, (21) and (22) formulate the classical spike train deconvolution problem that has been widely addressed in the literature [6-8].

Note that such a formulation can be extended to bidimensional data such as B-scan images. It can also be adapted to two-dimensional data in ultrasonic image restoration $[4,9]$ and SAFT [13-15].

\section{B. A linear model including attenuation and dispersion}

Let us consider the discrete-time signal $y_{n}=y\left(n \Delta_{t}\right)$, $n=0, \ldots, N_{y}-1$ where $\Delta_{t}=1 / f_{S}$ is the sampling period. From (21), one has:

$$
y_{n}=\int_{z} b(z) \int_{u} h_{e}(u) h_{a}\left(n \Delta_{t}-u-z / c_{0}, z\right) \mathrm{d} u \mathrm{~d} z .
$$

Discretizing the time integral at rate $\Delta_{t}$ yields:

$$
\begin{aligned}
y_{n} & \simeq \int_{z} b(z) \Delta_{t} \sum_{m} h_{e}\left(m \Delta_{t}\right) h_{a}\left((n-m) \Delta_{t}-\frac{z}{c_{0}}, z\right) \mathrm{d} z \\
& \simeq \Delta_{t} \sum_{m} h_{e}\left(m \Delta_{t}\right) \int_{z} b(z) h_{a}\left((n-m) \Delta_{t}-\frac{z}{c_{0}}, z\right) \mathrm{d} z .
\end{aligned}
$$

Note that using the data sampling period for the discretization rate is a practical choice that is commonly made in inverse problems. In particular, it yields a Toeplitz matrix structure that can be exploited for fast computations [48]. In [49], we have recently proposed a model with higher discretization rate, showing better estimation performance in some spike train deconvolution problems - but under the usual framework $h_{a}(t, z)=\delta(t)$. In this paper, we restrict the description to the discretization at rate $\Delta_{t}$ for the sake of clarity.

Similarly, the spatial integral is discretized at a given stepsize $\Delta_{z}$ :

$y_{n} \simeq \Delta_{t} \Delta_{z} \sum_{m} h_{e}\left(m \Delta_{t}\right) \sum_{i} b\left(i \Delta_{z}\right) h_{a}\left((n-m) \Delta_{t}-\frac{i \Delta_{z}}{c_{0}}, i\right.$

A natural choice is to consider $\Delta_{z}$ corresponding to the data time sampling $\Delta_{z}=c_{0} \Delta_{t}$. We then obtain:

$y_{n} \simeq \Delta_{t} \Delta_{z} \sum_{m} h_{e}\left(m \Delta_{t}\right) \sum_{i} b\left(i \Delta_{z}\right) h_{a}\left((n-m-i) \Delta_{t}, i \Delta_{z}\right)$

Let us now denote $x_{i}=\Delta_{t} \Delta_{z} b\left(i \Delta_{z}\right)$ and let us consider the column vectors $\boldsymbol{y}=\left[y_{0}, \ldots, y_{N_{y}-1}\right]^{T}$ and $\boldsymbol{x}=\left[x_{0}, \ldots, x_{N_{x}-1}\right]^{T}$, where superscript ${ }^{T}$ denotes matrix transposition. Note that $N_{x}$ and $N_{y}$ are not necessarily equal, depending on the boundary assumptions of the convolution [8]. We finally obtain the matrix-vector model:

$$
\boldsymbol{y}=\mathbf{H}_{e} \mathbf{H}_{a} \boldsymbol{x}+\boldsymbol{e}=\mathbf{G} \boldsymbol{x}+\boldsymbol{e},
$$

where:

- $\mathbf{H}_{e}$ is the convolution matrix corresponding to the instrumental response with elements $\left\{h_{e}\left((p-q) \Delta_{t}\right)\right\}_{p=0, \ldots, N_{y}-1, q=0, \ldots, N_{x}-1}, \quad$ where $p$ and $q$ respectively denote the line and the column indices. This matrix has a Toeplitz structure.

- $\mathbf{H}_{a}$ is the attenuation matrix with elements $\left\{h_{a}\left((n-i) \Delta_{t}, i \Delta_{z}\right)\right\}_{n=0, \ldots, N_{x}-1, i=0, \ldots, N_{x}-1}$. That is, the $i$-th column of $\mathbf{H}_{a}$ corresponds to the radiation impulse response at distance $z_{i}=i \Delta_{z}$. In our approach, it is computed from $H_{a}\left(f, i \Delta_{z}\right)$ in (11), with the power law attenuation model in (7) and the corresponding dispersive phase model defined by (14). In practice, $H_{a}(f, i \Delta z)$ is evaluated on a frequency grid in $\left[-f_{S} / 2, f_{S} / 2\right]$ with thin spacing such that temporal aliasing can be neglected. Then, the impulse response $\quad h_{a}((n-i) \Delta t, i \Delta z), n=0, \ldots, N_{x}-1 \quad$ is obtained by inverse discrete Fourier transform. Causality is imposed by setting $h_{a}((n-i) \Delta t, i \Delta z)=0$ for $n<i$. Consequently, $\mathbf{H}_{a}$ is lower triangular.

- $\mathbf{G}=\mathbf{H}_{e} \mathbf{H}_{a}$ combines the effects of the instrumental impulse responses and of the radiation impulse responses.

- $\boldsymbol{x}$ is the unknown sequence describing the target distribution.

- $\boldsymbol{e}$ is a perturbation term accounting for noise and model errors.

An example of matrices $\mathbf{H}_{e}, \mathbf{H}_{a}$ and $\mathbf{G}$ is given in Figure 7. The matrix $\mathbf{H}_{e}$ is built from the frontwall echo in Figure 6a. The matrix $\mathbf{H}_{a}$ is generated from the linear attenuation model $\alpha(f)=\alpha_{0}|f|$ and the dispersive relation defined in (14), with $\alpha_{0}=50 \mathrm{~Np} / \mathrm{MHz} / \mathrm{m}$. An example of data generated from the columns 200 and 1000 of the previous matrix $\mathbf{G}$ is also plotted in Figure 8. Note that attenuation provokes the widening of the second echo. Dispersion causes a phase distortion that creates a time shift of the echo envelope. Indeed, in $\mathbf{H}_{a}$, the maximum of each column is down-shifted away from the diagonal.

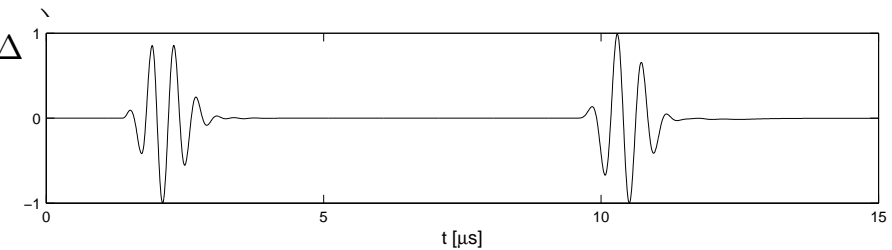

Fig. 8. Example of signal generated from the matrix $\mathbf{G}$ in Figure 7. The data $\boldsymbol{y}$ are computed from the columns 200 and 1000 of matrix $\mathbf{G}$ such that: $\boldsymbol{y}=\boldsymbol{g}_{200}+\boldsymbol{g}_{1000}$ where $\boldsymbol{g}_{i}$ is the $i$-th column of $\mathbf{G}$. Both echoes are normalized in amplitude for visualization clarity.

The model (27) states that data $\boldsymbol{y}$ are a noisy linear combination of columns of $\mathbf{G}$, and $\boldsymbol{x}$ collects the associated weights. Estimating $\boldsymbol{x}$ from $\boldsymbol{y}$ is an inverse problem that cannot be satisfactorily inverted in a least-squares sense: the matrix $\mathbf{G}$ is ill-conditioned and the generalized inverse $\left(\mathbf{G}^{T} \mathbf{G}\right)^{-1} \mathbf{G}^{T} \boldsymbol{y}$ hence suffers from uncontrolled noise amplification [8]. In the example above, the condition number of matrix $\mathbf{G}$ (which is a $1500 \times 1500$ matrix) is approximately $9.10^{19}$. 

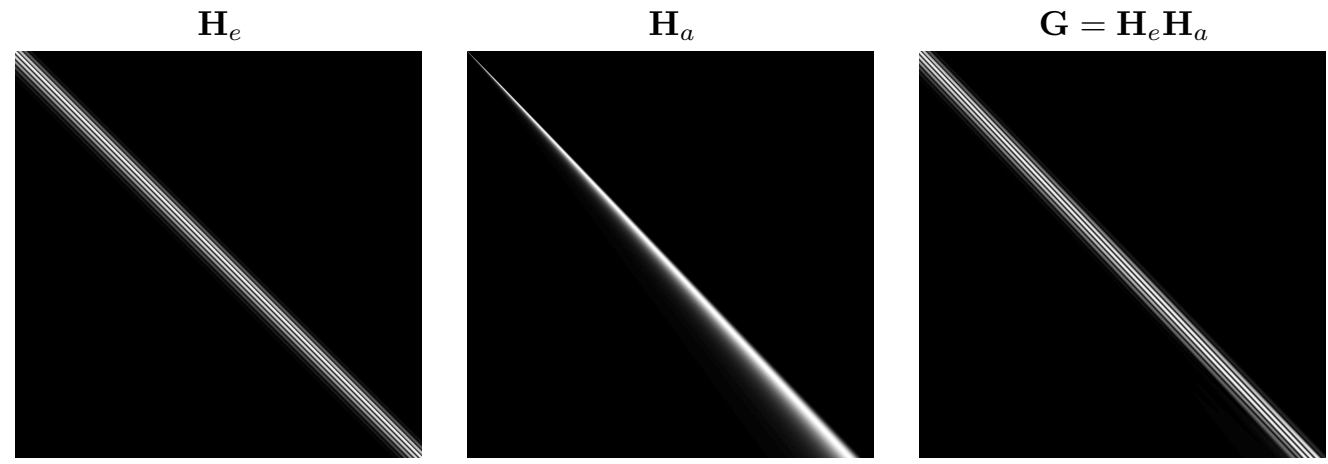

Fig. 7. Example of matrices $\mathbf{H}_{e}, \mathbf{H}_{a}$ and $\mathbf{G}$, where each column is normalised for representation clarity. $\mathbf{H}_{e}$ is the instrumental convolution matrix obtained from the frontwall echo in Figure 6 . In $\mathbf{H}_{a}$, each line corresponds to an instant $t_{n}=n \Delta_{t}$ and each column corresponds to a spatial distance $z_{i}=i \Delta_{z}$. $\mathbf{H}_{a}$ depends on both the attenuation and dispersion models. In this example, $\alpha(f)=\alpha_{0}|f|$ with $\alpha_{0}=50 \mathrm{~Np} / \mathrm{MHz} / \mathrm{m}$. The dispersion is set using the model in (14). The matrix $\mathbf{G}$ combines both instrumental and attenuative effects.

\section{Comparison with Olofsson's model}

From (5), the radiation transfer function at $z_{i}=i \Delta_{z}$ reads:

$$
\begin{aligned}
H_{r}\left(f, z_{i}\right) & =b\left(z_{i}\right) e^{-[\alpha(f)+j \beta(f)] i \Delta_{z}} \\
& =b\left(z_{i}\right)\left[e^{-(\alpha(f)+j \beta(f)) \Delta z}\right]^{i} \\
& =b\left(z_{i}\right) P(f)^{i} .
\end{aligned}
$$

It corresponds, up to a multiplicative constant, to the $i$-th power of the frequency kernel $P(f)=e^{-(\alpha(f)+j \beta(f)) \Delta z}$. Let $\rho_{n}, n=0, \ldots, N-1$ represent the discrete-time sequence with Fourier transform $P(f)$ for $f \in\left[-f_{S} / 2, f_{S} / 2\right]$. It represents the radiation impulse response between two elementary spatial layers, separated by $\Delta_{z}$. Equation (28) states that the radiation impulse response at $z_{i}$ is equal to $i-1$ self-convolutions of the time kernel $\rho$.

In a time-domain setting, Olofsson and Stepinski [7] proposed an empirical choice for the kernel $\rho$, defined by:

$$
\rho_{0}=0, \rho_{1}=1-a, \rho_{2}=a \text { and } \rho_{n}=0 \quad \forall n \geq 3,
$$

with $a>0$ and close to zero. Such a kernel implies a small distortion between two elementary layers spaced by $\Delta_{z}$ and is hence close to a delayed Kronecker delta function. Because of its first zero, this kernel generates causal impulse responses in $\mathbf{H}_{a}$. The transfer function between two elementary layers consequently behaves as a low-pass filter:

$$
|P(f)|^{2}=1-4 a(1-a) \sin ^{2}\left(\pi \Delta_{t} f\right) .
$$

The corresponding attenuation function can be found by:

$$
\alpha(f)=-\frac{\ln |P(f)|}{\Delta_{z}} .
$$

Such a model has shown satisfactory results in a deconvolution context [7]. However, it seems less accurate in terms of physical reality, since $\alpha(f)$ is more adequately modeled by a frequency power law. In addition, the tuning of parameter $a$ looks somehow arbitrary, whereas frequency-based models can be tuned according to physical models, or even set from a material catalog [47]. An example of radiation transfer functions $\left|H_{r}(f, z)\right|$ for Olofsson's model is plotted in Figure $3 \mathrm{~b}$ for $a=0.2$. As it will be shown in IV-C, this value yields a model which can be compared to the linear attenuation model with $\alpha_{0}=50 \mathrm{~Np} / \mathrm{MHz} / \mathrm{m}$ at propagation distance $z=10 \mathrm{~mm}$. However, the two attenuation models yield quite different radiation transfer functions for other distances, as shows the comparison between Figures $3 \mathrm{a}$ and $3 \mathrm{~b}$. The performances of frequencybased models and of Olofsson's model are compared for basic experimental data in the following section.

\section{Model EXPloitation In THE CASE OF A SINGle ECHO: ESTIMATION OF MATERIAL PARAMETERS}

\section{A. Single echo detection by "matched filtering"}

We consider data made up of two well-separated echoes in a configuration similar to the one of Figures 5 and 6 . The instrumental response $h_{e}$ is identified from the frontwall echo, from which the matrix $\mathbf{H}_{e}$ in (27) is built. The matrix $\mathbf{H}_{a}$ depends on the considered attenuation model. Identifying the backwall echo then amounts to selecting the column of matrix $\boldsymbol{G}$, say $\boldsymbol{g}_{\hat{\imath}}$, that best fits the data ${ }^{2}$. That is, $\boldsymbol{x}$ should have only one non-zero coefficient at index $\hat{\imath}$ that corresponds to the spatial position $z_{\hat{\imath}}=\hat{\imath} \Delta_{z}$ of the back surface. Consider the minimization of the least-squares misfit criterion between the data and the model:

$$
\left(\hat{\imath}, \hat{x}_{\hat{\imath}}\right)=\underset{i=0, \ldots, N_{x}-1, x_{i} \in \mathbb{R}}{\arg \min }\left\|\boldsymbol{y}-\boldsymbol{g}_{i} x_{i}\right\|^{2},
$$

which statistically corresponds to the maximum likelihood estimation of the one-column model $\boldsymbol{y}=\boldsymbol{g}_{i} x_{i}+\boldsymbol{e}$ under the assumption that the noise samples in $e$ are independently, identically and normally distributed [8]. As this problem is linear in $x_{i}$, the best $\hat{x}_{i}$ can be found for a given $\boldsymbol{g}_{i}$ by:

$$
\hat{x}_{i}=\frac{\boldsymbol{g}_{i}^{T} \boldsymbol{y}}{\left\|\boldsymbol{g}_{i}\right\|^{2}} .
$$

Inserting (33) into (32), after simple manipulations, the optimal position $\hat{\imath}$ is:

$$
\hat{\imath}=\underset{i=0, \ldots, N_{x}-1}{\arg \max } \frac{\left|\boldsymbol{g}_{i}^{T} \boldsymbol{y}\right|}{\left\|\boldsymbol{g}_{i}\right\|^{2}} .
$$

\footnotetext{
${ }^{2}$ In practice, the contribution of the frontwall echo is previously removed from the data.
} 
Finally, the estimated echo is obtained by $\hat{\boldsymbol{y}}=\boldsymbol{g}_{\hat{\imath}} \hat{x}_{\hat{\imath}}$. Such a procedure follows a matched filtering approach in that it selects the column in $\mathbf{G}$ that yields the maximum correlation with data $\boldsymbol{y}$ - up to a normalization term. However, it is not strictly speaking a matched filter since $\mathbf{G}$ is not a convolution matrix due to the spatially-variant nature of the attenuation matrix $\mathbf{H}_{a}$.

\section{B. Joint estimation of the attenuation parameter and sound} velocity

For a frequency power law attenuation model $\alpha(f)=\alpha_{0}|f|^{\gamma}$ with given $\gamma$, the previous echo detection procedure enables the joint estimation of the attenuation parameter $\alpha_{0}$ and the reference velocity $c_{0}$, which are physical quantities of interest for a given material. Indeed, the attenuation transfer function in (11) at distance $z_{i}=i \Delta_{z}$ reads:

$$
\begin{aligned}
H_{a}\left(f, z_{i}\right) & =e^{-[\alpha(f)+j \mathcal{H}(\alpha(f))] i \Delta_{z}} \\
& =e^{-\left[|f|^{\gamma}+j \mathcal{H}\left(|f|^{\gamma}\right)\right] \alpha_{0} c_{0} i \Delta_{t}} .
\end{aligned}
$$

Hence, for a given $\gamma$, the model (11) only depends on the single parameter $\chi_{0}=\alpha_{0} c_{0}$. For each $\chi_{0}$, the velocity $c_{0}$ can be deduced from the optimal position $\hat{\imath}$, found by the matched filter as in (34), and from the thickness of the plate $d$ by:

$$
c_{0}=\frac{2 d}{\hat{\imath} \Delta_{t}} .
$$

The associated attenuation parameter is then given by $\alpha_{0}=$ $\chi_{0} / c_{0}$. As mentioned in II-B, $c_{0}$ is not the group velocity but the phase velocity for $f=\infty$. Note that standard non destructive evaluation (NDE) methods generally rely on the estimation of the group velocity because dispersion is not considered.

In practice, we can apply the matched filtering procedure for different values of $\chi_{0}$ in an arbitrarily thin grid, and select the best value $\widehat{\chi}_{0}$ that minimizes the residue between the data and the model: $r=\|\boldsymbol{y}-\hat{\boldsymbol{y}}\| /\|\boldsymbol{y}\|$. This value consequently leads to $\widehat{c}_{0}$ and $\widehat{\alpha}_{0}$ that are the best estimations in a least-squares sense of those material quantities for a given propagation model. This procedure can be used for NDE which aims at estimating the material properties. However, it can only be applied if the frontwall and the backwall echoes are wellseparated.

Note that if the mechanical properties of the material are known, $c_{0}$ can be obtained from the analytic formula of sound speed of longitudinal waves [1]:

$$
c_{0}=\sqrt{\frac{E(1-\nu)}{\rho(1+\nu)(1-2 \nu)}},
$$

with $E$ the Young's modulus, $\nu$ the Poisson's ratio and $\rho$ the density.

\section{Application to polycarbonate}

We apply the method described above to estimate the attenuation and velocity parameters of an homogeneous material. The same measurement configuration as in II-D is designed with a $2.25 \mathrm{MHz}$ center frequency transducer used in pulseecho mode. We use a clear polycarbonate plate of thickness $\mathrm{e}=10.2 \mathrm{~mm}$, known to have linear attenuation $(\gamma=1)$ and to be highly attenuative $[5,47]$. The thickness is measured with a digital caliper, with precision $\pm 0.1 \mathrm{~mm}$, accounting for both instrument imprecision and irregularities of the plate.

We compare the estimations obtained with the following propagation models:

1) without attenuation

2) with linear attenuation and linear phase

3) with linear attenuation and dispersive phase

4) Olofsson's model

For Olofsson's model, we select parameter $a$ in (29) that best fits the echo. The corresponding matched filtering procedure also yields an estimation of $c_{0}$ - see (36) - but it does not provide any estimation of $\alpha_{0}$. The residue values of the estimated backwall echoes are displayed in Figures 9a and 9b, as a function of $\chi_{0}$ for linear attenuation models and of $a$ for Olofsson's model, respectively.

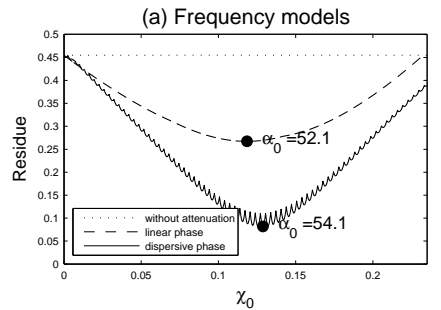

(c) Without attenuation

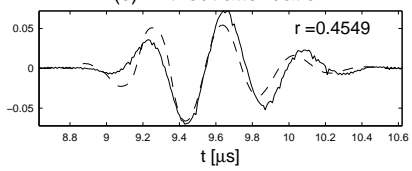

(e) Dispersive phase

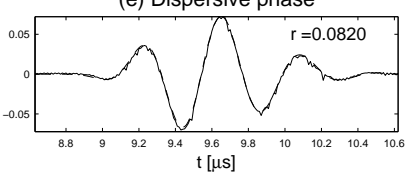

(b) Olofsson model

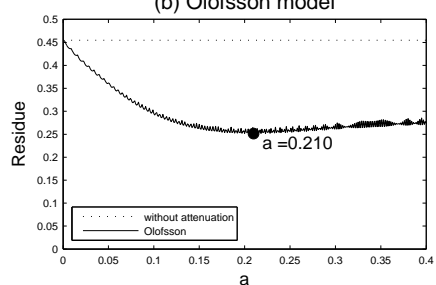

(d) Linear phase

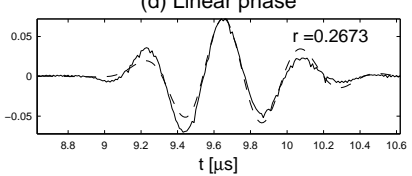

(f) Olofsson

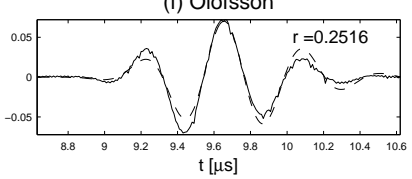

Fig. 9. Matched-filtering-based backwall echo estimation from a polycarbonate plate of thickness $d=10.2 \mathrm{~mm}$ using a $2.25 \mathrm{MHz}$ transducer. (a): Residue of estimation as a function of the parameter $\chi_{0}$, for several models of linear attenuation (without attenuation, linear phase, dispersive phase). (b): Residue of estimation as a function of the parameter $a$ for Olofsson's model. (c-d-e-f): Data $\boldsymbol{y}$ (solid line) and estimation $\hat{\boldsymbol{y}}$ (dashed line) with the optimal attenuation parameters, (c) without attenuation, (d) with linear phase, (e) with dispersive phase and (f) with Olofsson's model ( $r$ is the residue).

One can clearly see that the dispersive model produces the lowest residue $(r \sim 0.08)$. The linear phase model and Olofsson's model lead to approximately the same residue, $r \sim 0.27$ and $r \sim 0.25$ respectively. The estimated waveform without attenuation shows the greatest discrepancy with the data $(r \sim 0.45)$. Because of the poor adequacy of the model, the matched filter returns a positive amplitude $\widehat{x}_{\hat{\imath}}$ whereas a negative value is expected. Indeed the reflection coefficient between polycarbonate and water is negative [46]. As a consequence, a better estimated waveform should be leftshifted by half the wave cycle. The corresponding values of $\widehat{c}_{0}$ and $\widehat{\alpha}_{0}$ are listed in Table I. The attenuation parameter given by the dispersive model is $54.1 \mathrm{~Np} / \mathrm{MHz} / \mathrm{m}$, and the one obtained 


\begin{tabular}{|c|c|c|c|c|c|}
\hline & & $\widehat{\chi}_{0}$ & $\hat{\imath} \Delta_{t}(\mu \mathrm{s})$ & $\widehat{c}_{0}(\mathrm{~m} / \mathrm{s})$ & $\widehat{\alpha}_{0}(\mathrm{~Np} / \mathrm{MHz} / \mathrm{m})$ \\
\hline 1 & Without att. & & 8.76 & 2329 & \\
\hline 2 & Lin. phase & 0.118 & 8.97 & 2274 & 52.1 \\
\hline 3 & Disp. phase & 0.129 & 8.57 & 2380 & 54.1 \\
\hline 4 & Olofsson & & 7.42 & 2749 & \\
\hline
\end{tabular}

ESTIMATED $\widehat{\chi}_{0}$, TIMES OF FLIGHT, REFERENCE VELOCITIES AND ATTENUATION COEFFICIENTS FOR A 10.2 MM-THICK PLATE OF POLYCARBONATE USING A 2.25 MHZ PROBE. FOR MODELS 2 AND 3, $\gamma=1$.

from the linear phase model is $52.1 \mathrm{~Np} / \mathrm{MHz} / \mathrm{m}$. Both are in the range of the values reported in the literature (for example, $[50,57] \mathrm{Np} / \mathrm{MHz} / \mathrm{m}$ in [47]). We consider the value given by the dispersive model as more likely as this model yields the lowest estimation residual. The optimal attenuation parameter of Olofsson's model is 0.210 but it cannot be linked to any reference value.

As expected, the linear phase model that considers a constant phase velocity $c(f)$ leads to $\widehat{c}_{0}=2274 \mathrm{~m} / \mathrm{s}$ very close to the reference group velocity given in [47]: $2270 \mathrm{~m} / \mathrm{s}$. The value returned by the dispersive model, $2380 \mathrm{~m} / \mathrm{s}$, is logically larger than the group velocity, since it corresponds to the phase velocity at infinite frequency (see II-B). Olofsson's model leads to $2749 \mathrm{~m} / \mathrm{s}$, that is difficult to interpret. The inaccurate modeling obtained with the non-attenuated waveform leads to an estimated velocity of $2329 \mathrm{~m} / \mathrm{s}$, whereas it should return the same value as the linear phase model since both models consider constant phase velocity.

In this study, the dispersive model gives accurate results for polycarbonate, for which frequency-dependent attenuation can be well represented by a linear model. Such an approach could also be applied to non-linear attenuation models with given $\gamma$, e.g., for synthetic rubber and castor oil [40].

\section{Application to THE DECONVOLUtion OF NDT DATA: ESTIMATION OF THE THICKNESS OF A PLATE}

In this section, an inverse problem of spike train deconvolution is considered. From a pulse-echo measurement, our goal is to estimate the thickness of the polycarbonate plate that was already used in section IV-C. More precisely, we consider the problem of estimating a spike train $\boldsymbol{x}$ from data $\boldsymbol{y}=\mathbf{G} \boldsymbol{x}+\boldsymbol{e}$, where the locations of the spikes - the non-zero elements in vector $\boldsymbol{x}$ - correspond to the positions of the echoes. The distance between consecutive spikes then corresponds to the plate thickness.

Such a framework can be applied to a large variety of practical NDT problems [1], in order to measure the thickness of a layer or a wall. Applications occur for the manufacture of pipes, plates, strips, etc. and for the control of walls in severe environments (power plants, chemical industry), when the back area is out of reach. In such cases, a pulse-echo acquisition may be an appropriate solution. Note that, in the presented example, the echoes are well separated, hence the deconvolution problem is not complex from an informational point of view. In the case of thinner layers, the reflected echoes overlap and advanced processing methods such as deconvolution are appropriate tools to estimate the positions of the echoes.
Here, we use a transducer with $5 \mathrm{MHz}$ center-frequency. Compared to the $2.25 \mathrm{MHz}$ transducer used in the previous experiment, better resolution is expected but the attenuation effect is stronger. The backwall echo is strongly distorted and highly attenuated in amplitude, with a very low signal-tonoise ratio (see Figures 10a and 10b). A direct time-of-flight identification by visual inspection might lead to inaccurate thickness estimation because of the low signal to noise ratio and of the phase shift.
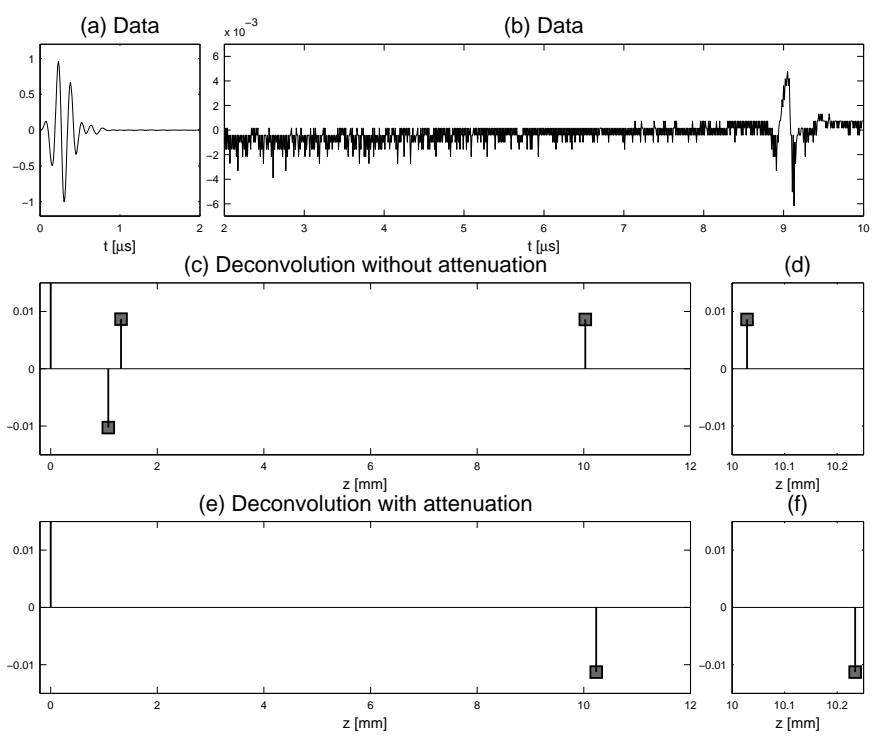

Fig. 10. Deconvolution of data acquired from a polycarbonate plate of thickness $\mathrm{d}=10.2 \mathrm{~mm}$ with a $5 \mathrm{MHz}$ transducer. (a) Data for $t \in[0,2] \mu \mathrm{s}$ corresponding to the frontwall echo, (b) data for $t \in[2,10] \mu \mathrm{s}$ where a zoom in amplitude is performed. (c-d): deconvolution without attenuation model $\left(c_{0}=2274 \mathrm{~m} / \mathrm{s}\right)$. (e-f): deconvolution with linear attenuation and dispersive model $\left(c_{0}=2380 \mathrm{~m} / \mathrm{s}, \gamma=1, \alpha_{0}=54.1 \mathrm{~Np} / \mathrm{MHz} / \mathrm{m}\right)$.

Deconvolution is performed through the minimization of the least-squares data misfit function, penalized by the $\ell_{1}$ norm $\|\boldsymbol{x}\|_{1}=\sum_{i}\left|x_{i}\right|$ :

$$
\hat{\boldsymbol{x}}=\underset{\boldsymbol{x}}{\arg \min }\|\boldsymbol{y}-\mathbf{G} \boldsymbol{x}\|^{2}+\lambda\|\boldsymbol{x}\|_{1} .
$$

Such a sparsity-inducing penalization produces few non-zero elements in the solution for appropriate $\lambda$. Criterion (38) is a convex function and admits a unique minimum for a given $\lambda$. The regularization parameter $\lambda$ introduces a tradeoff between the least-squares fit and the penalization. The value $\lambda=0$ corresponds to the least-squares solution, that is not acceptable due to the ill-posedness of the problem [8]. Then, as $\lambda$ increases, the number of spikes in $\boldsymbol{x}$ tends to decrease. This kind of formulation has shown a great interest in inverse problems in the past decades [50], and particularly in ultrasonic deconvolution problems $[6,8]$. Optimization is performed using the homotopy continuation method described in [51]. In this part, $\lambda$ is set manually in order to retrieve one spike in the area of $10 \mathrm{~mm}$.

We consider deconvolution using the attenuation-free model and the model with attenuation and dispersion. The first model is the approach commonly adopted in deconvolution problems. The matrix $\mathbf{G}$ is equal to $\mathbf{H}_{e}$, that is built from the frontwall echo shown in Figure 10a. The second model 
includes $\mathbf{G}=\mathbf{H}_{e} \mathbf{H}_{a}$ where $\mathbf{H}_{a}$ is built from the dispersive model (11). Its parameters are set from the experimental results in Table I. We have seen in IV-C that the estimation of $c_{0}$ for the model without attenuation was erroneous. Hence, for this model, we set $c_{0}$ to the most plausible estimated group velocity, that is $2274 \mathrm{~m} / \mathrm{s}$. Note that the sampling frequency is $100 \mathrm{MHz}$, leading to the spatial precision $\Delta_{z}=c_{0} \Delta_{t}=0.0274 \mathrm{~mm}$ and $0.0238 \mathrm{~mm}$ for the two models, respectively. According to [46], the true sequence is composed of a positive spike at $0 \mathrm{~mm}$ and of a negative spike at $10.2 \mathrm{~mm}$.

Results are shown in panels c-f of Figure 10. Deconvolution with the attenuation-free model estimates four spikes. They correspond to the frontwall position, two false detections and the backwall position. The last spike has a positive amplitude, which is inconsistent with the truth (as in IV-C). The corresponding thickness estimation is $10.5 \mathrm{~mm}$. As shown in subplots e-f, the result using attenuation and dispersion in the model shows two spikes, that correspond to the frontwall and the backwall positions. They have a positive and a negative sign respectively, which is coherent with the expected object. The estimated distance is $10.23 \mathrm{~mm}$, that is closer to the measured value. Using the model with attenuation and dispersion in criterion (38) achieves more accurate spike detection than the standard convolution model with $\mathbf{G}=\mathbf{H}_{e}$, and hence leads to more satisfactory thickness estimation.

\section{DISCUSSION AND FUTURE WORKS}

We have presented a discrete model of ultrasonic signals that considers attenuation and dispersion. The model is built in the frequency domain, where specific radiation transfer functions are computed for a finite set of distances. We obtain a linear model between the data and the unknown spatial distribution of targets. Compared to the usual formulation, it amounts to introducing an attenuation matrix depending on acoustic propagation parameters. Experimental results from attenuative materials reveal the accuracy of such a formulation for the modeling of the backscattered echoes. Better results are obtained compared to the non-causal model that considers constant phase velocity [3] and to the causal empirical model proposed in [7]. The proposed model also yields the best results on the problem of detecting and locating the backwall echo in a polycarbonate plate. Finally, we consider a spike train deconvolution problem based on $\ell_{1}$-norm regularization in order to estimate the thickness of a plate. With the proposed model, the backwall echo blurred into noise is successfully located whereas the solution based on the standard model shows false detections and an imprecise echo location.

Future works could concern the application of the developed model to more complex data, in particular to the deconvolution of A-scans with overlapping echoes. In such cases, the better adequacy of the proposed model should improve the spike detection performance compared to standard deconvolution approaches using the generic, stationary, convolution model. We also expect that algorithms based on such a model can yield better performance than parametric models [23,24], which also allow some flexibility in the echo shapes but do not integrate any constraint due to the ultrasound propagation properties.
Similar models for ultrasonic data could also be developed for two and three-dimensional acquisitions. In particular, our approach could be extended for modeling SAFT [13,14] and Full Matrix Capture [15] data in ultrasonic imaging.

We have seen that our model depends on a reference velocity, which corresponds to the phase velocity at infinite frequency. It can be obtained from the mechanical properties of the material. We have also shown that it can be estimated from a material evaluation process. In addition, complementary works could link such reference velocity to the group velocity, inspired by Gurumurthy and Arthur [32] who proposed an empirical relation between the two quantities.

Finally, our model considers a single geometrical signature of the acoustical targets, which is particularly appropriate if the targets have the same shape, as for plane surfaces in the presented experiments.

Future works could include the diffraction of typical reflectors (Flat Bottom Holes for instance) by considering different possible signatures. In such an approach, the attenuation matrix would be replaced by a set of matrices, each one characterizing a specific diffraction signature. We believe that such dictionary-based model, coupled with efficient sparsityaware algorithms [50], may be appropriate to address complex NDT problems for the detection and the characterization of flaws. A similar approach could be used to detect cracks or delaminations in multilayered materials. Indeed, a set of several attenuation matrices can also describe the different paths produced by multiple reflections.

\section{ACKNOWLEDGEMENTS}

The authors would like to thank Aroune Duclos from the University of Maine in Le Mans, France, for the help provided to perform the ultrasonic measurements and for helpful discussions about ultrasonic propagation. We also wish to acknowledge Vincent Baltazart from IFSTTAR in Nantes, France, for valuable discussions about Q-filtering.

We are also grateful to the reviewers for their critical reading and valuable comments.

This work has been partially supported by the French Région Pays de la Loire as part of the scientific program "Non-Destructive Testing and Evaluation - Pays de la Loire" (ECND-PdL).

\section{APPENDIX}

\section{EXPRESSION OF THE DISPERSIVE PHASE WITH LINEAR} ATTENUATION

With linear attenuation $\alpha(f)=\alpha_{0}|f|$, the phase term (15) reads $\mathcal{H}(\alpha(f))=-\frac{\alpha_{0} f_{S}}{4 \pi^{2}} \mathcal{J}\left(\frac{2 \pi f}{f_{S}}\right)$, with:

$$
\mathcal{J}(\omega)=\mathcal{P} \int_{-\pi}^{\pi}|v| \cot \left(\frac{\omega-v}{2}\right) \mathrm{d} v, \omega \in[-\pi, \pi] .
$$

Function $\mathcal{J}(\omega)$ is odd with $\mathcal{J}(\pi)=0$. Consider $\omega \in] 0, \pi[$. With $u=\omega-v$, one has:

$$
\mathcal{J}(\omega)=\mathcal{P} \int_{\omega-\pi}^{\omega}(\omega-u) \cot \frac{u}{2} \mathrm{~d} u-\int_{\omega}^{\omega+\pi}(\omega-u) \cot \frac{u}{2} \mathrm{~d} u,
$$


where the Cauchy principal value of the first integral excludes 0 from its domain. Let $F_{\omega}(u)$ be an antiderivative of $(\omega-u) \cot \frac{u}{2}$. An antiderivative of $\cot \frac{u}{2}$ is $2 \ln \left|\sin \frac{u}{2}\right|$. Hence integration by parts yields for any $u \in]-2 \pi, 2 \pi[, u \neq 0$ :

$$
F_{\omega}(u)=2(\omega-u) \ln \left|\sin \frac{u}{2}\right|+2 \int_{0}^{u} \ln \left|\sin \frac{\varphi}{2}\right| \mathrm{d} \varphi .
$$

The last integral also reads:

$$
\int_{0}^{u} \ln \left|\sin \frac{\varphi}{2}\right| \mathrm{d} \varphi=-\mathrm{Cl}_{2}(u)-u \ln (2),
$$

where $\mathrm{Cl}_{2}(u)=-\int_{0}^{u} \ln \left|2 \sin \frac{\varphi}{2}\right| \mathrm{d} \varphi$ is the Clausen function of order 2, i.e., the imaginary part of the dilogarithm of $e^{j u}$ [52]. From (40)-(42), one can show that:

$$
\begin{aligned}
\mathcal{J}(\omega) & =2 \mathrm{Cl}_{2}(\omega+\pi)+2 \mathrm{Cl}_{2}(\omega-\pi)-4 \mathrm{Cl}_{2}(\omega) \\
& =4\left(\mathrm{Cl}_{2}(\omega+\pi)-\mathrm{Cl}_{2}(\omega)\right) .
\end{aligned}
$$

Finally, it can be shown that (43) also holds for $w \in[-\pi, 0]$.

\section{REFERENCES}

[1] J. Krautkramer and H. Krautkramer, Ultrasonic Testing of materials. Berlin: Springer-Verlag, 1990.

[2] S.-K. Sin and C.-H. Chen, "A comparison of deconvolution techniques for the ultrasonic nondestructive evaluation of materials," IEEE Transactions on Image Processing, vol. 1, pp. 3-10, January 1992.

[3] A. C. Kak and K. A. Dines, "Signal processing of broadband pulsed ultrasound: Measurement of attenuation of soft biological tissues," IEEE Transactions on Biomedical Engineering, vol. 25, pp. 321-344, July 1978.

[4] M. Fatemi and A. C. Kak, "Ultrasonic B-scan imaging: Theory of image formation and a technique for restoration," Ultrasonic Imaging, vol. 2, pp. 1-47, January 1980.

[5] P. Droin, G. Berger, and P. Laugier, "Velocity dispersion of acoustic waves in cancellous bone," IEEE Transactions on Ultrasonics, Ferroelectrics and Frequency Control, vol. 45, pp. 581-592, May 1998.

[6] M. S. O'Brien, A. N. Sinclair, and S. M. Kramer, "Recovery of a sparse spike time series by L1 norm deconvolution," IEEE Transactions on Signal Processing, vol. 42, pp. 3353-3365, December 1994.

[7] T. Olofsson and T. Stepinski, "Minimum entropy deconvolution of pulseecho signals acquired from attenuative layered media," The Journal of the Acoustical Society of America, vol. 109, pp. 2831-2839, June 2001.

[8] J. Idier, Bayesian Approach to Inverse Problems. ISTE Ltd and John Wiley \& Sons Inc, April 2008.

[9] J. A. Jensen, "Estimation of pulses in ultrasound B-scan images," IEEE Transactions on Medical Imaging, vol. 10, pp. 164-172, June 1991.

[10] J. Greenleaf, S. Johnson, S. Lee, G. Hermant, and E. Woo, "Algebraic reconstruction of spatial distributions of acoustic absorption within tissue from their two-dimensional acoustic projections," in Acoustical Holography (P. Green, ed.), pp. 591-603, Springer US, 1974.

[11] G. Glover and J. C. Sharp, "Reconstruction of ultrasound propagation speed distributions in soft tissue: Time-Of-Flight tomography," IEEE Transactions on Sonics and Ultrasonics, vol. 24, pp. 229-234, July 1977.

[12] J. Greenleaf and R. Bahn, "Clinical imaging with transmissive ultrasonic computerized tomography," IEEE Transactions on Biomedical Engineering, vol. 28, pp. 177-185, February 1981.

[13] M. Karaman, L. Pai-Chi, and M. O'donnell, "Synthetic aperture imaging for small scale systems," IEEE Transactions on Ultrasonics, Ferroelectrics and Frequency Control, vol. 42, pp. 429-442, May 1995.

[14] F. Lingvall, T. Olofsson, and T. Stepinski, "Synthetic aperture imaging using sources with finite aperture: Deconvolution of the spatial impulse response," The Journal of the Acoustical Society of America, vol. 114, pp. 225-234, July 2003.

[15] C. Holmes, B. W. Drinkwater, and P. Wilcox, "Post-processing of the full matrix of ultrasonic transmit-receive array data for non-destructive evaluation," NDT\&E International, vol. 38, pp. 701-711, December 2005.

[16] P. A. Narayana and J. Ophir, "A closed form method for the measurement of attenuation in nonlinearly dispersive media," Ultrasonic Imaging, vol. 5, pp. 117-21, January 1983.
[17] K. Wear, "The effects of frequency-dependent attenuation and dispersion on sound speed measurements: applications in human trabecular bone," IEEE Transactions on Ultrasonics, Ferroelectrics and Frequency Control, vol. 47, pp. 265-273, January 2000.

[18] G. Haiat, F. Padilla, R. Cleveland, and P. Laugier, "Effects of frequencydependent attenuation and velocity dispersion on in vitro ultrasound velocity measurements in intact human femur specimens," IEEE Transactions on Ultrasonics, Ferroelectrics and Frequency Control, vol. 53, pp. 39-51, January 2006.

[19] D. Hale, "An inverse Q-filter," Stanford Exploration Project, vol. 26, pp. 231-244, 1981

[20] J. M. Mendel, Optimal Seismic Deconvolution: an Estimation Based Approach. New York: Academic Press, 1983.

[21] Y. Wang, Seismic Inverse Q-filtering. ISTE Ltd and John Wiley \& Sons Inc, January 2009.

[22] E. Kjartansson, "Constant Q-Wave Propagation and Attenuation," Journal of Geophysical Research, vol. 84, pp. 4737-4748, August 1979.

[23] R. Demirli and J. Saniie, "Model-based estimation of ultrasonic echoes. Part I: Analysis and algorithms," IEEE Transactions on Ultrasonics, Ferroelectrics and Frequency Control, vol. 48, pp. 787-802, May 2001.

[24] Y. Lu and J. Michaels, "Numerical implementation of matching pursuit for the analysis of complex ultrasonic signals," IEEE Transactions on Ultrasonics, Ferroelectrics and Frequency Control, vol. 55, pp. 173-182, January 2008.

[25] G.-M. Zhang, D. M. Harvey, and D. R. Braden, "Signal denoising and ultrasonic flaw detection via overcomplete and sparse representations," The Journal of the Acoustical Society of America, vol. 124, pp. 29632972, November 2008.

[26] E. Mor, A. Azoulay, and M. Aladjem, "A matching pursuit method for approximating overlapping ultrasonic echoes," IEEE Transactions on Ultrasonics, Ferroelectrics and Frequency Control, vol. 57, pp. 19962004, September 2010.

[27] K. Kaaresen and E. Bolviken, "Blind deconvolution of ultrasonic traces accounting for pulse variance," IEEE Transactions on Ultrasonics, Ferroelectrics and Frequency Control, vol. 46, pp. 564-573, May 1999.

[28] K. Rasmussen, "Maximum likelihood estimation of the attenuated ultrasound pulse," IEEE Transactions on Signal Processing, vol. 42, pp. 220-222, January 1994.

[29] M. Fink and J.-F. Cardoso, "Diffraction effects in pulse-echo measurement," IEEE Transactions on Sonics and Ultrasonics, vol. 31, pp. 313329, July 1984.

[30] P. R. Stephanishen, "Transient radiation from pistons in an infinite baffle," The Journal of American Society of America, vol. 49, no. 5, pp. 1629-1638, 1971.

[31] M. O’Donnell, E. T. Jaynes, and J. G. Miller, "General relationships between ultrasonic attenuation and dispersion," The Journal of the Acoustical Society of America, vol. 63, pp. 1935-1937, June 1978.

[32] K. Gurumurthy and R. Arthur, "A dispersive model for the propagation of ultrasound in soft tissue," Ultrasonic Imaging, vol. 4, pp. 355-377, October 1982

[33] C. Lee, M. Lahham, and B. Martin, "Experimental verification of the Kramers-Kronig relationship for acoustic waves," IEEE Transactions on Ultrasonics, Ferroelectrics and Frequency Control, vol. 37, pp. 286-294, July 1990.

[34] T. Szabo, "Causal theories and data for acoustic attenuation obeying a frequency power law," The Journal of the Acoustical Society of America, vol. 97, pp. 14-24, January 1995.

[35] R. Kuc, "Generating a minimum-phase digital filter model for the acoustic attenuation of soft tissue," in Ultrasonics Symposium, pp. 794 796, October 1983.

[36] R. Kuc, "Modeling acoustic attenuation of soft tissue with a minimumphase filter," Ultrasonic Imaging, vol. 6, pp. 24-36, January 1984.

[37] C.-H. Chen, W.-L. Hsu, and S.-K. Sin, "A comparison of wavelet deconvolution techniques for ultrasonic NDT," in International Conference on Acoustics, Speech, and Signal Processing, vol. 2, (New-York, USA), pp. 867-870, April 1988.

[38] A. Lhémery, "Impulse-response method to predict echo-responses from targets of complex geometry. Part I: Theory," The Journal of the Acoustical Society of America, vol. 90, pp. 2799-2807, November 1991.

[39] F. A. Duck, Physical properties of tissue. London: Academic Press, 1990.

[40] P. He, "Experimental verification of models for determining dispersion from attenuation," IEEE Transactions on Ultrasonics, Ferroelectrics and Frequency Control, vol. 46, pp. 706-714, May 1999.

[41] J. Ophir and P. Jaeger, "Spectral shifts of ultrasonic propagation through media with nonlinear dispersive attenuation," Ultrasonic Imaging, vol. 4 pp. 282-289, July 1982. 
[42] J. F. Kelly, R. J. McGough, and M. M. Meerschaert, "Analytical timedomain Green's functions for power-law media," The Journal of the Acoustical Society of America, vol. 124, pp. 2861-2872, November 2008.

[43] T. Szabo, "Time domain wave equations for lossy media obeying a frequency power law," The Journal of the Acoustical Society of America, vol. 96, pp. 491-500, July 1994.

[44] A. Papoulis, The Fourier integral and its applications. McGraw-Hill electronic sciences series, McGraw-Hill, 1962.

[45] A. Oppenheim and R. Schafer, Discrete-time signal processing. PrenticeHall signal processing series, Prentice Hall, 1989.

[46] J. Saniie and D. Nagle, "Pattern recognition in the ultrasonic imaging of reverberant multilayered structures," IEEE Transactions on Ultrasonics, Ferroelectrics and Frequency Control, vol. 36, pp. 80-92, January 1989.

[47] A. Selfridge, "Approximate material properties in isotropic materials," IEEE Transactions on Sonics and Ultrasonics, vol. 32, pp. 381-394, May 1985.

[48] G. Golub and C. Van Loan, Matrix Computations, vol. 3. Baltimore and London: Johns Hopkins University Press, October 1996.

[49] E. Carcreff, S. Bourguignon, J. Idier, and L. Simon, "Resolution enhancement of ultrasonic signals by up-sampled sparse deconvolution," in Proceedings of the IEEE International Conference on Acoustic, Speech and Signal Processing, (Vancouver, Canada), May 2013.

[50] J. Tropp and S. Wright, "Computational methods for sparse solution of linear inverse problems," Proceedings of the IEEE, vol. 98, pp. 948-958, June 2010.

[51] D. M. Malioutov, M. Cetin, and A. S. Willsky, "Homotopy continuation for sparse signal representation," in IEEE International Conference on Acoustics, Speech, and Signal Processing, vol. 5, (Philadelphia, USA), pp. 733-736, March 2005.

[52] L. C. Maximon, "The dilogarithm function for complex argument," Proceedings of the Royal Society of London. Series A: Mathematical, Physical and Engineering Sciences, vol. 459, pp. 2807-2819, November 2003. 